\title{
Stimulation Effects of SmartBox for E-learning Using JXTA-Overlay P2P System
}

\author{
Keita Matsuo $\nmid$, Leonard Barolli $\nmid$, Vladi Kolici $\dagger \dagger$, Fatos Xhafa $\nmid \dagger$, \\ Akio Koyama ${ }^{\ddagger \dagger}$, Arjan Durresi ${ }^{\ddagger \ddagger}$ \\ $\dagger$ Graduate School of Engineering, Fukuoka Institute of Technology (FIT) \\ 3-30-1 Wajiro-Higashi, Higashi-Ku, 811-0295 Fukuoka, Japan \\ E-mail: bd07002@bene.fit.ac.jp \\ $\ddagger$ Department of Information and Communication Engineering \\ Fukuoka Institute of Technology (FIT) \\ 3-30-1 Wajiro-Higashi, Higashi-Ku, 811-0295 Fukuoka, Japan \\ E-mail: barolli@ fit.ac.jp \\ $\dagger †$ Department of Electronics and Telecommunication \\ Polytechnic University of Tirana \\ Mother Teresa Square, Nr.4, Tirana, Albania \\ ladi@istitech.net \\ $\dagger \ddagger$ Department of Languages and Informatics Systems \\ Polytechnic University of Catalonia \\ Jordi Girona 1-3, 08034 Barcelona, Spain \\ E-mail: fatos@lsi.upc.edu \\ ${ }^{\ddagger}$ Department of Informatics, Yamagata University \\ 4-3-16 Jonan, Yonezawa 992-8510, Yamagata, Japan \\ E-mail: akoyama@yz.yamagata-u.ac.jp \\ ${ }_{\ddagger}^{\ddagger}$ Department of Computer and Information Science \\ Indiana University Purdue University at Indianapolis (IUPUI) \\ 723 W. Michigan Street SL 280, Indianapolis, IN 46202, USA \\ E-mail: durresi@cs.iupui.edu
}

\begin{abstract}
This work is motivated by the need to develop decentralized P2P approaches to support learning and teaching activity in virtual universities. In this paper, we present the stimulation effects of the SmartBox for e-learning using JXTA-Overlay P2P System. We also show the design and implementation of the SmartBox environment that is used for stimulating the learners motivation to increase the learning efficiency. The SmartBox is integrated with our P2P system as a useful tool for monitoring and controlling learners' activity. We found by experimental results that the SmartBox is an effective way to increase the learner's concentration. We also investigated the relation between learner's body movement, concentration, and amount of study. We found that when the learner is solving more exercises he has more body movements. This shows that when the number of body movements is hight, the learner's concentration is high. We also consider the feasibility of our P2P platform to integrate different computational end-devices.
\end{abstract}

\section{Introduction}

In the past few years we have observed an explosive growth of multimedia computing, communication and applications. Information Technology (IT) has a great effect in our everyday life and is transforming the way people live, work, and interact with each other, and is impacting the way businesses, government services, education, entertainment, and health care are operating.

The Internet is growing every day and the performance of computers is increased exponentially. However, the Internet architecture is based on Client/Server (C/S) topology, therefore can not use efficiently the clients features. It should be noted that computers of today have the same characteristics with conventional super-computers. Also, with appearance of new technologies such as ad-hoc networks, sensor networks, body networks, home networking, new network devices and application will appear. Therefore, it is very important to monitor, control and optimize these network devices via communication channels. However, in large- 
scale networks such as Internet, it is very difficult to control the network devices, because of the security problems. Each network has its own security policy and the information should overcome the firewalls.

The firewalls are used for checking the signal information between private and public networks. The signals are transmitted according to some decided rules and it is very difficult to change the network security policy. So, there are many small networks and Intranets that do not allow the information coming from other networks. This happens especially in the networks of schools or libraries. Therefore, recently many researchers are working on Peer-to-Peer (P2P) networks, which are able to overcome the firewalls, NATs and other security devices without changing the network policy. Therefore, $\mathrm{P} 2 \mathrm{P}$ architectures will be very important for future e-learning systems.

Virtual campuses and virtual universities are new ways of organizing students activities by using IT technologies. The virtual university is one of the most widely used form of virtual organizations in the today's teaching and learning activities. However, most of the current virtual organizations use server-mediated Internet-based applications that enable sharing among the members of the community.

In $\mathrm{C} / \mathrm{S}$ approaches the shared resources are centralized on servers and members of the virtual community (students, teachers, researchers, etc.) access them through the Web clients. Thus, every thing is done at server side while at the client side just an interface is needed. In general, the server-mediated approaches show several drawbacks, especially lack of scalability and efficiency in virtual campuses of many thousands of students.

The most powerful alternative to server-based approaches for applications of virtual universities is the use of decentralized approaches such as P2P systems. In such systems, the computational burden of the system can be distributed to peer nodes of the system. Therefore, in decentralized systems users become themselves actors by sharing, contributing and controlling the resources of the systems. This characteristic makes $\mathrm{P} 2 \mathrm{P}$ systems very interesting for the development of decentralized applications $[1,2]$ for virtual campuses and virtual organizations.

During the last decade, many e-learning systems are proposed and implemented, mostly using web technologies. With emerging of new technologies such as P2P, sensor and ad-hoc networks, it will be possible to build ubiquitous systems that can offer an equal educational environment regardless the region, age, time or the place [3, 4].

This work is motivated by the need to develop decentralized $\mathrm{P} 2 \mathrm{P}$ approaches to support learning and teaching activity in virtual universities. In this work, we present the design and implementation of main features of a JXTA-based P2P system (called JXTA-Overlay) that support in many ways the development of e-learning applications. JXTA-Overlay is thus a platform for developing group-ware applications. We also show the implementation and the design of a SmartBox environment that will be used for stimulating the learn- ers to increase the learning efficiency. The SmartBox is integrated with the JXTA-Overlay by using efficient message sending between peers in the system.

The structure of this paper is as follows. In Section 2, we introduce the related work. In Section 3, we introduce the Project JXTA and explain JXTA-Overlay. In Section 4, we present the proposed $\mathrm{P} 2 \mathrm{P}$ e-learning system. In section 5, we present experimental result of the SmartBox's functions Finally, conclusions and future work are given in Section 6.

\section{Related Work}

In this section, we discuss the related work for P2P systems and Web based e-learning systems.

Much of the initial research efforts and projects on $\mathrm{P} 2 \mathrm{P}$ systems were concerned with the investigation of overlay networks. It should be noted that each time more, this effort is oriented towards the design and implementation of libraries and platforms to support the development of real P2P applications, which could actually combine both Grid and P2P technologies. Bal et al. [5] motivated the need for research from conceptual, algorithmic and application level tools to facilitate the application development task, that is, tools that enable writing, deploying and running Grid/P2P applications.

Since in P2P applications file sharing plays an important role, a lot of research effort is being devoted to the development of reliable file sharing systems to be used in P2P applications [6]. In particular, several studies are done for measuring the efficiency in $\mathrm{P} 2 \mathrm{P}$ systems $[7,8]$ as it is crucial for such systems.

Due to the opportunities provided by the Internet, more and more people are taking advantage of online distance learning courses. During the last few years enormous research efforts have been dedicated to the development of elearning systems. Consequently, many large projects have been established $[9,10,11,12,13,14]$. However, in these systems the e-learning completion rate is low. One of the reasons is the low study interest when the learner studies the subjects. Therefore, it is very important to stimulate learner's motivation during the study. In our previous works $[15,16]$, we proposed an agent based distance learning system to deliver appropriate studying materials to learners and stimulate learner's motivation.

However, all these systems are based on $\mathrm{C} / \mathrm{S}$ architecture. In such approach the shared resources are centralized on servers and members of the virtual community (clients) access them through request protocols. Every thing is done at server side while at the client side just an interface is needed. Among the most important limitations of server-mediated approaches, we could distinguish lack of scalability, lack of fault tolerance, usually low performance/bottlenecks as well as high costs in acquiring, developing and maintaining such applications. 


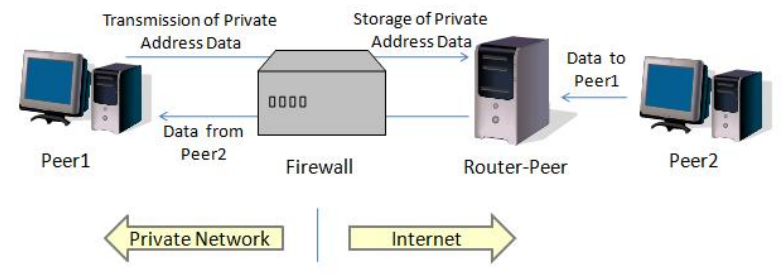

Figure 1. P2P communication.

\section{JXTA Technology and JXTA-Overlay}

\subsection{JXTA Technology}

JXTA technology is a generalized group of protocols that allow different devices to communicate and collaborate among them. JXTA offers a platform covering basic needs in developing P2P networks [17].

By using JXTA framework is possible that a peer in a private network can be connected to a peer in the Internet by overcoming existing Firewalls as shown in Fig. 1. The procedure to overcome the fire-wall is as follows.

- In the Router Peer is stored the private address of Peer 1 by using the HTTP protocol to pass the firewall from Peer1.

- The Router Peer receives the data from Peer2 and access the Private address of Peer 1 to transmit the data.

JXTA is an interesting alternative for developing P2P systems and groupware tools to support online teams of students in virtual campuses. In particular, it is appropriate for file sharing given that its protocols allow to develop either pure or mixed $\mathrm{P} 2 \mathrm{P}$ networks. This last property is certainly important since pure $\mathrm{P} 2 \mathrm{P}$ systems need not the presence of a server for managing the network.

\subsection{JXTA-Overlay}

JXTA-Overlay project is an effort to use JXTA technology for building an overlay on top of JXTA offering a set of basic primitives (functionalities) that are most commonly needed in JXTA-based applications. The proposed overlay comprises the following primitives:

- Peer discovery,

- Peer's resources discovery,

- Resource allocation,

- Task submission and execution,

- File/data sharing, discovery and transmission,

- Instant communication,

- Peer group functionalities (groups, rooms),

- Monitoring of peers, groups and tasks.

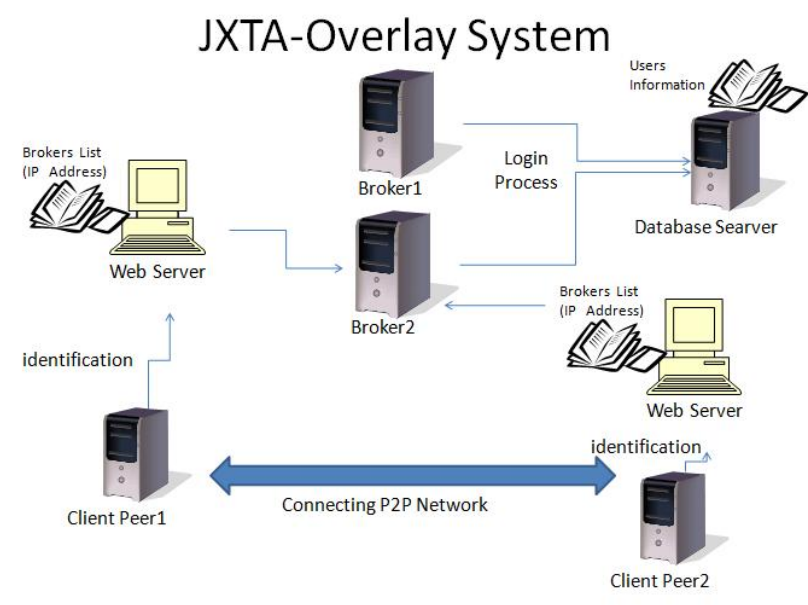

Figure 2. Structure of JXTA-Overlay system.

This set of basic functionalities is intended to be as complete as possible to satisfy the needs of JXTA-based applications. The overlay is built on top of JXTA layer and provides a set of primitives that can be used by other applications, which on their hand, will be built on top of the overlay, with complete independence. The JXTA-Overlay project has been developed using the latest updated JXTA libraries. In fact, the project offers several improvements of the original JXTA protocols/services, on the one hand, to increase the reliability of JXTA-based distributed applications and to support group management and file sharing [1].

The architecture of the $\mathrm{P} 2 \mathrm{P}$ distributed platform we have developed using JXTA technology has these building blocks: Broker Module, Primitives Module and Client Module. Altogether these three modules form a new overlay on top of JXTA. The structure of JXTA-Overlay system is shown in Fig. 2.

\section{Proposed P2P e-Learning System}

\subsection{Transmission Control and Management in JXTA-Overlay}

The most important part in e-learning systems or virtual campus is the communication between peers (groups of students) or between teachers and students. By using the proposed JXTA-Overlay, it is possible to overcome, firewalls, routers, NATs, and bridges in the private networks. We explain in following the message transmission by the JXTA protocol.

JXTA protocol uses Universally Unique Identifier (UUID) in order to identify the peers in the private network from the Internet. The UUID is a general unique identifier. It is generated by the NIC address of the computer, date and time. By knowing the UUID and TCP address, it is possible to make address translation. 


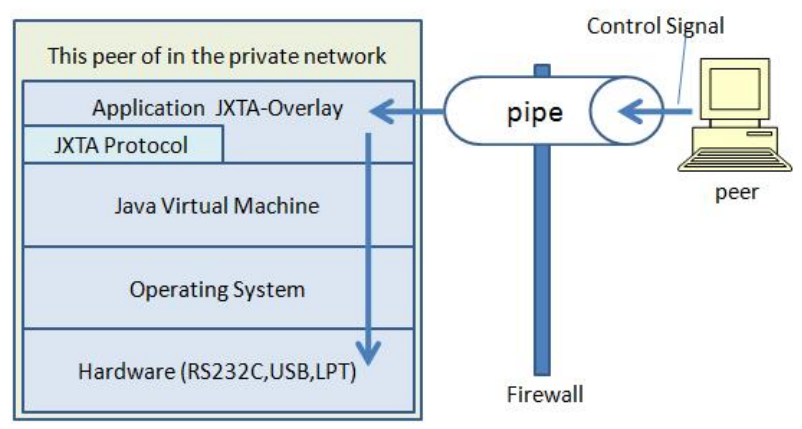

Figure 3. Implementation of remote control in JXTA-Overlay.

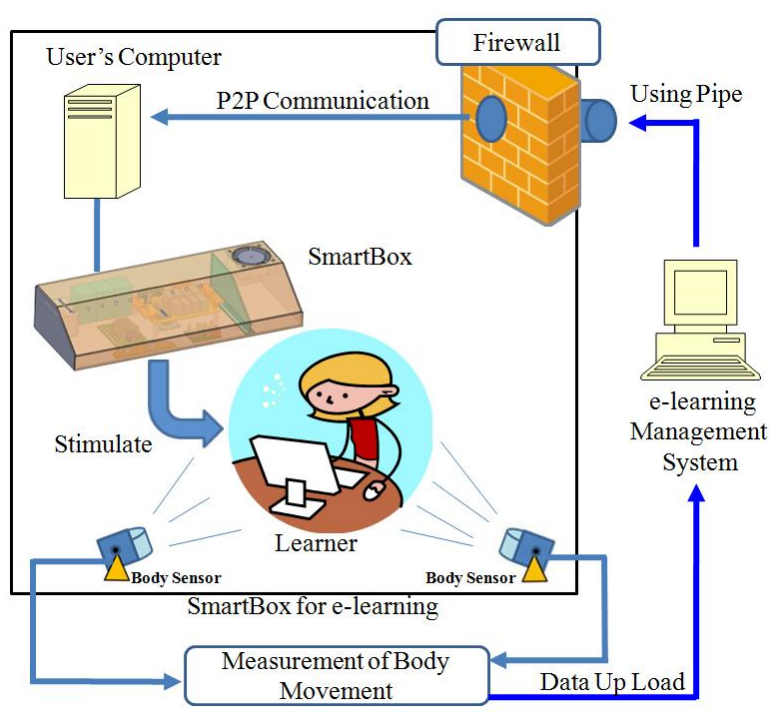

\section{Figure 4. Proposed P2P e-learning system structure.}

We implemented a control system that is able to control a peer in a private network from a peer in the Internet as showing in Fig. 3. The control targets are considered the network devices such as RS232C port, LPT port and USB port. By implementing this kind of control system, we are able to collect data and control the peers in a wide area network. Thus, we will be able to control all devices that are connected to the peers. We control the USB devices and RS232C equipment. This is because USB devices are very popular and are used almost in every computer. Also, by using USB is possible to control the motors and LEDs. The RS232C is legal interface and many devices have implemented it.

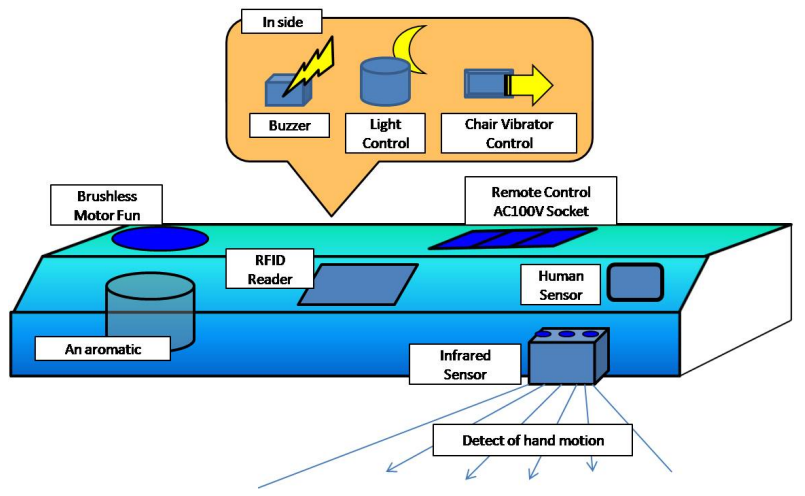

Figure 5. SmartBox functions.

\subsection{SmartBox Design, Implementation and Inte- gration with P2P System}

In our proposed system we combine P2P, Web and Sensor technologies. We propose a P2P e-learning system as shown in Fig. 4. Our goal is not only to monitor and control the students' activity in a wide area network but also to stimulate and increase learners' motivation. For this reason, we proposed and implemented a SmartBox as shown in Fig. 5.

Previously, we implemented a SmartBox device which was big in size and difficult to be moved in different places. The size of previous SmartBox was $50 \times 10 \times 15 \mathrm{~cm}$. In order to occupy a small space in the learner's desk and to transport easily in different places we designed and implemented a new SmartBox. The size of new SmartBox is $35 \times 7 \times 12$ $\mathrm{cm}$. The SmartBox is equipped with different sensors (for sensing learner situation) and devices (used for stimulating learner's motivation).

The SmartBox has the following sensors and functions.

- Body Sensor: for detecting the learner's body movement.

- Chair Vibrator Control: for vibrating the learner's chair.

- Light Control: for adjusting the room light for study.

- Smell Control: for controlling the room smell.

- Sound Control: to emit relaxing sounds.

- Remote Control Socket: for controlling AC 100V socket (on-off control).

A snapshot of the previous SmartBox and new SmartBox is shown in Fig. 6. The use of SmartBox for e-learning is shown in Fig. 7. The SmarBox can detect the learners body movement by using body sensor. 


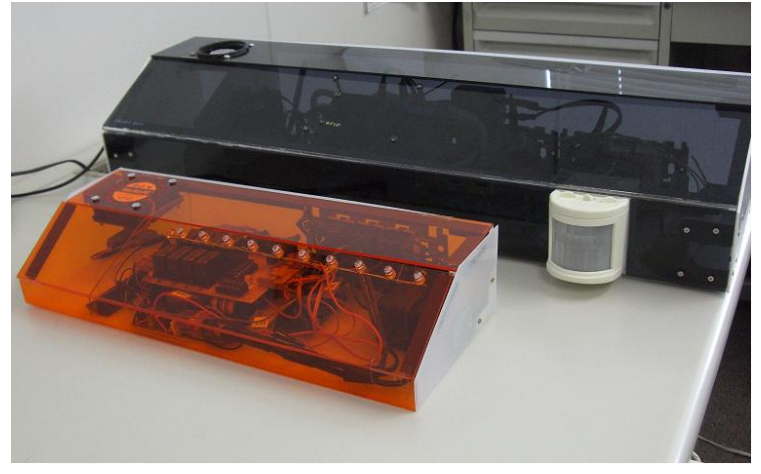

Figure 6. A snapshot of new SmartBox (Front) and previous SmartBox (Back).

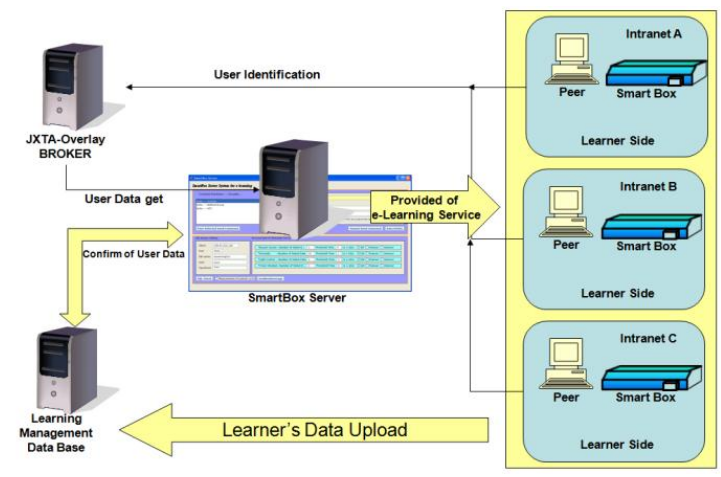

Figure 7. System structure when SmartBox is used for e-learning.

\section{Experimental Results}

\subsection{Body and Hand Movement}

The proposed system can detect the learner's movement by using body sensors. The measurement data for learners' body movements are shown in Fig. 8. We got these data after observing two learners studying for 40 hours. After a sensor reacts, the interval time will be reset to zero. We consider where is the point of effective stimulation for learners from the sensing rate values. We pay attention to the reaction frequency of sensor. The interval of sensors response is very active at the point around 20-30 seconds, but it gets less active after 30 seconds. This tells us that the learners make certain actions once every 30 seconds while they are studying. After 50 seconds, there is almost no reaction from the sensor. We show in Fig. 9 the sensor response range middle value and the average response time. Moreover, we can obtain the stimulation point by using the polynomial approximation curve.

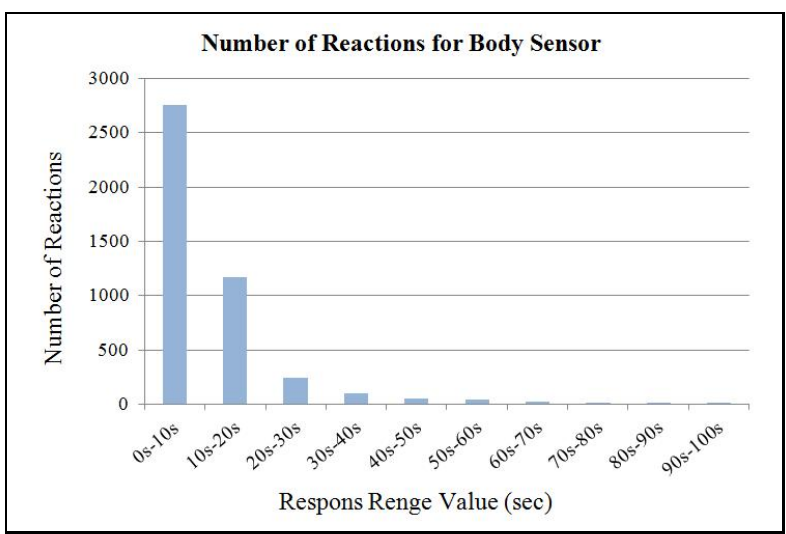

Figure 8. Reaction number for body sensor.

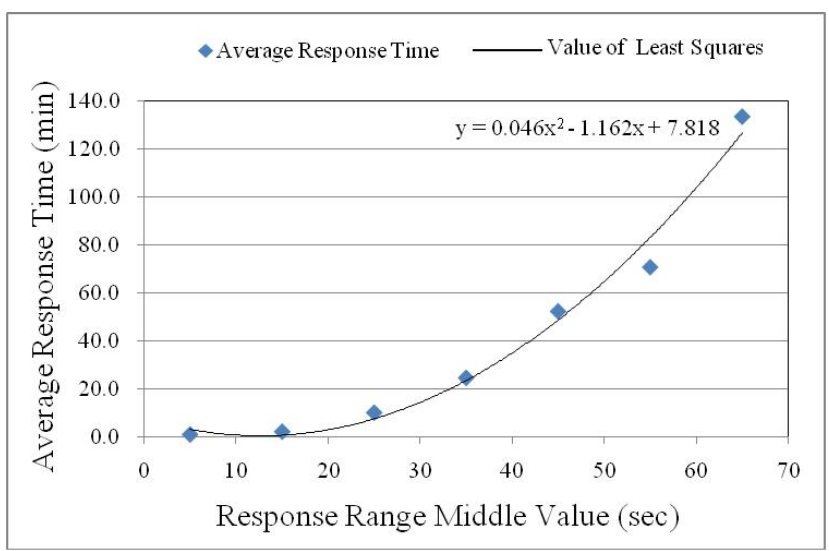

Figure 9. Experimental results and polynomial approximation curve.

\subsection{Evaluation of SmartBox Functions}

In our experiments, we used SmartBox and measured the stimulation effects that the SmartBox has to the learners. We checked the Smell function, Light function (high luminance LED) and Sound function (different kind of music). In order to evaluate the effects of SmartBox on the learners, we used human sensor of SmartBox and retrieved the information of the body movement. Using this information, we activated the following functions.

- Aroma Function

- Light Brightness Function

- Sound and Buzzer Function 


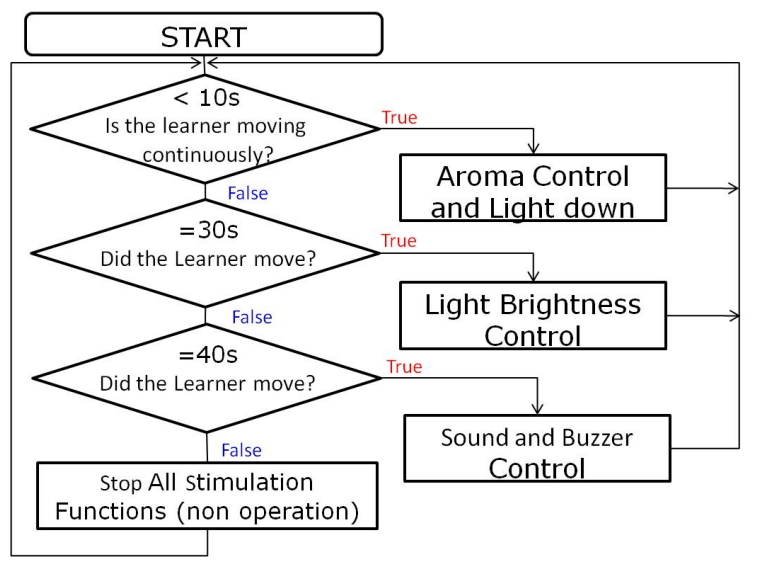

Figure 10. Flowchart of learner stimulation.

The flowchart of learner's stimulation is shown in Fig. 10. When the system detects the body movement within $10 \mathrm{sec}-$ onds, the system activates the Aroma Function. If the learners did not move for about 30 seconds, we should give them a slight stimulation by light. In the case when the learner did not move for about 40 seconds from the start, the system gives the sound stimulation.

\subsection{Measurement of Learner's Body Movement During Study}

In Fig. 11, we show the measurement of the learner body in the case when the learner looses the concentration. The graph shows that the learner is sleeping and there is not movement of his body or hands, because the response time has a higher value.

In order to check the effects of the SmartBox on the learner, we carried out two experiments. In these experiments, a high school student is studying English and Mathematics. In Fig. 12 is shown the case when the learner used the SmartBox, while in Fig. 13 the case when the user did not use the SmartBox. As shown in Fig. 12, when the movement of learner's body is not detected for 30 seconds, we gave to the learner a stimulation by light (High Brightness LED). At that time, the learner is regaining the concentration. Also, if the learner did not move for about 40 seconds, we gave the sound stimulation. The comparison between these two figures shows that the use of SmartBox is an effective way to improve the learner motivation, because the learner's concentration is higher using the SmartBox.

\subsection{Relation Between Learner's Movement Body, Concentration, and Amount of Study}

For the investigation of the movement of learner's body, concentration and amount of study, we used a Japanese Drill Text Book that has 100 exercises in each page as shown in Fig. 14. The reasons for this selection are as follows.

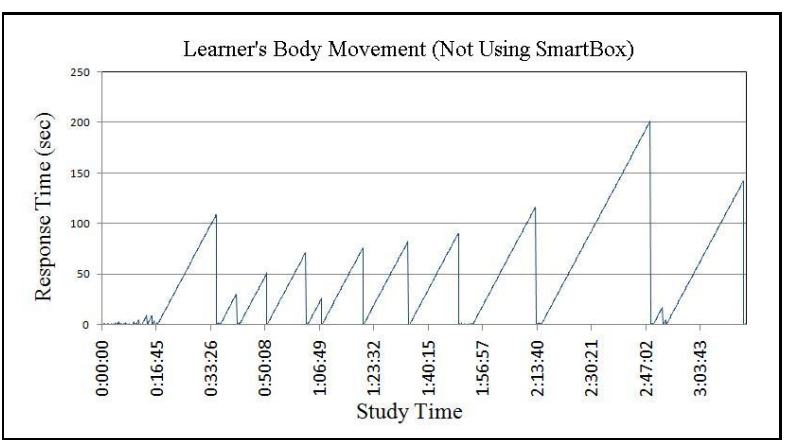

Figure 11. A case when student looses concentration.

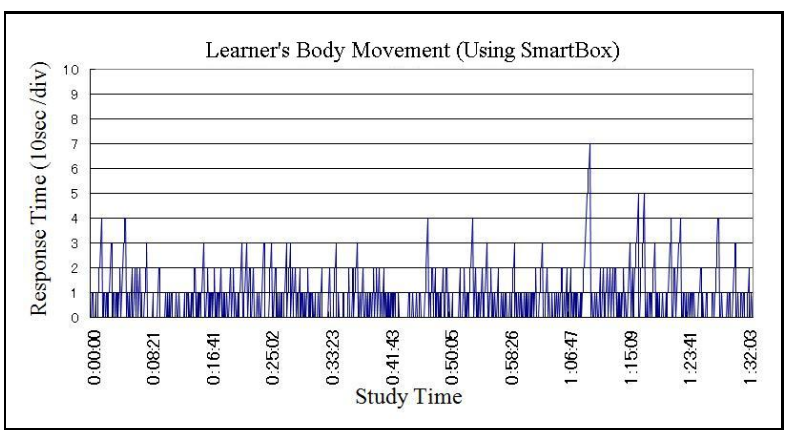

Figure 12. Measurement of high school student concentration using SmartBox.

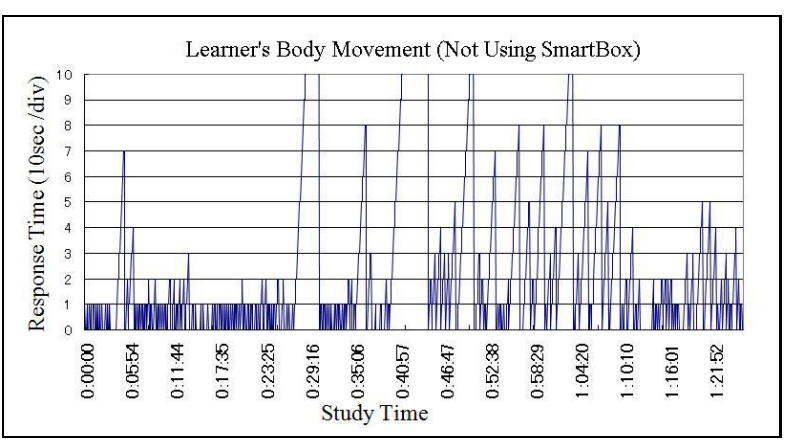

Figure 13. Measurement of high school student concentration without using SmartBox.

- It is monotonous study work and it is difficult for the learner to maintain concentration for a long time.

- It is easy for expressing the amount of study numerically. 


\begin{tabular}{|c|c|c|c|c|c|c|c|c|c|c|c|}
\hline \hline+ & $\mathbf{9}$ & $\mathbf{4}$ & $\mathbf{5}$ & $\mathbf{8}$ & $\mathbf{1 0}$ & $\mathbf{1}$ & $\mathbf{2}$ & $\mathbf{3}$ & $\mathbf{7}$ & $\mathbf{6}$ \\
\hline $\mathbf{4}$ & 13 & & & & & & & & & \\
\hline $\mathbf{5}$ & & & & 13 & & & 7 & & & \\
\hline $\mathbf{2}$ & & & & & & & & & & 8 \\
\hline $\mathbf{6}$ & & & 11 & & & & & & & \\
\hline $\mathbf{3}$ & & & & & & 4 & & & & \\
\hline $\mathbf{9}$ & & & & 17 & & & & & & \\
\hline $\mathbf{1 0}$ & & & & & & & 12 & & & \\
\hline $\mathbf{8}$ & & & & & & & & & 15 & \\
\hline $\mathbf{1}$ & & 5 & & & & & & & & \\
\hline $\mathbf{7}$ & & & & & & 8 & & 10 & & \\
\hline
\end{tabular}

Figure 14. Learner's text book with 100 exercises in each page.

- Everyone can solve these exercises regardless of individual knowledge or experience.

We considered as a subject, a fifth grade pupil. By studying everyday, he becomes accustomed to this text book. In Fig. 15, we show the experimental results when the pupil used the SmartBox. The experimental results show that the learner's concentration is high. We carried out another experiment with the same study subject as shown in Fig. 16. The experimental results indicate that the learner's concentration is increased gradually. Especially, after about 20 minutes from the start of study the concentration is very high. Comparing Fig. 15 with Fig. 16, we conclude that number of body movements or the response time is smaller when there are more exercises to be solved. Also, the concentration can be increased in the case when the system detects the body movement in a short cycle.

In Fig. 17, we show the experimental results when the pupil did not use the SmartBox. The experimental results show that the learner's concentration is low.

The comparison of experimental results in Fig. 15, Fig. 16 and Fig. 17 for the amount of study is shown in Table 1. The amount of study is the number of exercises for minutes. From Table 1 results can be seen that the learner is solving more problems (exercises) when he has more body movements. This shows that when the number of body movements is hight, the learner's concentration is high.

From the experimental results, we conclude that the use of SmartBox is an effective way to stimulate the learners in order to continue studying while maintaining the concentration.

\section{Conclusions and Future Work}

In this work, we presented the evaluation of SmartBox for e-learning using JXTA-Overlay P2P System. The proposed system is able to stimulate the learners in a wide area network by using JXTA-Overlay system. The SmartBox is

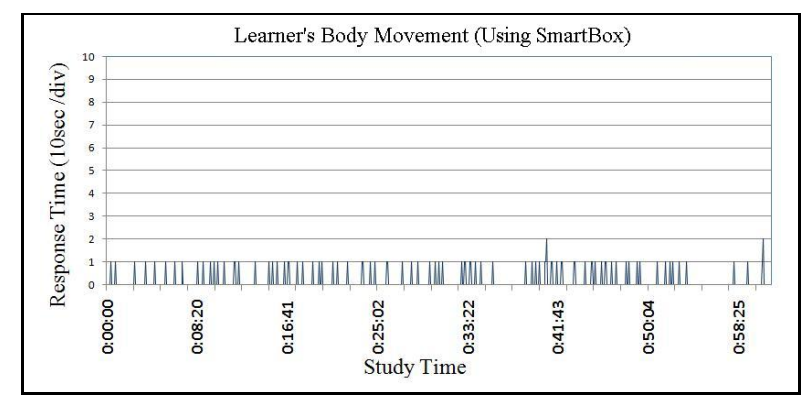

Figure 15. First experiment with elementary school pupil using SmartBox.

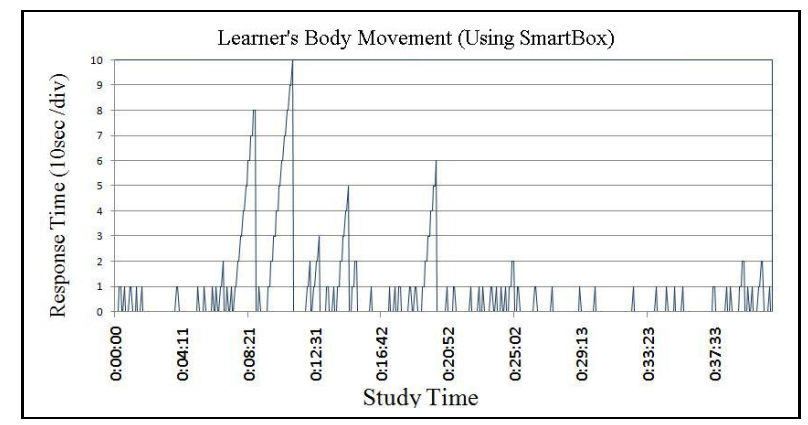

Figure 16. Second experiment with elementary school pupil using SmartBox.

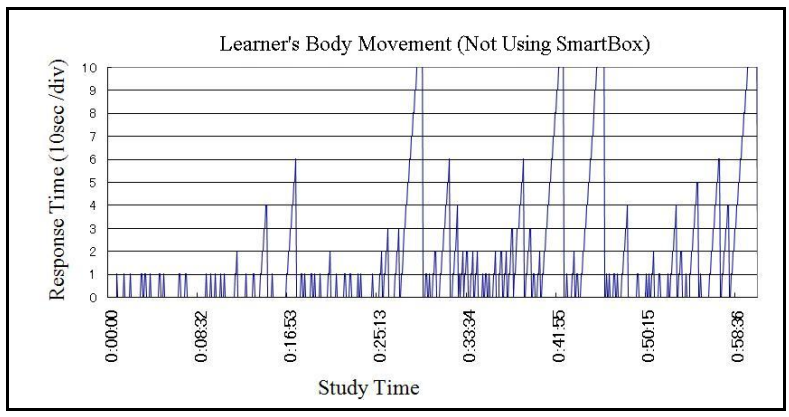

Figure 17. Experimental results with elementary school pupil without using SmartBox.

integrated with the $\mathrm{P} 2 \mathrm{P}$ system by benefiting features of $\mathrm{P} 2 \mathrm{P}$ systems such as direct communication among peers.

We carried out many experimental results to evaluate the effects of SmartBox for e-learning. From the simulation results, we concluded as follows.

- The use of SmartBox is an effective way to increase the learner's concentration.

- We derived the relation between learner's body movement, concentration, and amount of study.

- The learner is solving more exercises when his body has more movements. This shows that when the num- 
Table 1. Relation between learner's body movement, concentration, and amount of study.

\begin{tabular}{lcc}
\hline Figure No. & Body Movement & Amount of Study \\
\hline Fig. 15 & High & $21.31 / \mathrm{min}$ \\
Fig. 16 & Middle & $14.45 / \mathrm{min}$ \\
Fig. 17 & Low & $5.20 / \mathrm{min}$ \\
\hline
\end{tabular}

ber of body movements is hight, the learner's concentration is high.

In the future, we plan to evaluate the proposed system for many learners. We also would like to evaluate the proposed system for different scenarios where monitoring and control of students in the wide area is important.

\section{References}

[1] F. Xhafa, R. Fernandez, T. Daradoumis, L. Barolli, S. Caballe, "Improvement of JXTA Protocols for Supporting Reliable Distributed Applications in P2P Systems", Proc. of NBiS-2007 (Regensburg, Germany), LNCS 4658, pp.345-354, September 2007.

[2] L. Barolli, F. Xhafa, A. Durresi, G. De Marco, "M3PS: A JXTA-based Multi-platform P2P System and Its Web Application Tools", International Journal of Web Information Systems, Vol. 2, No. 3/4, pp. 187-196, 2006.

[3] IBM Corporation, "Making P2P Interoperable: Creating JXTA Systems Design P2P Systems that Extend Beyond Traditional Network Boundaries", 2007.

[4] Sun Microsystems, "JXTA Java Standard Edition v2.5: Programmers Guide", June 2007.

[5] H. Bal, H. Casanova, J. Dongarra, and S. Matsuoka, "Application-Level Tools", In Foster et al., eds, The Grid: Blueprint for a New Computing Infrastructure, Chapter 24, pp. 463-489, Morgan Kaufmann, 2003.

[6] S. M. Lui and S.H. Kwok, "Interoperability of Peer-toPeer File Sharing Protocols", SIGecom Exch.Journal, Vol. 3, No. 3, pp. 25-33, ACM Press, New York, NY, USA, 2002.

[7] F. Le Fessant, S. Handurukande, A. M. Kermarrec, L. Massouli, "Clustering in Peer-to-Peer File Sharing Workloads", Peer-to-Peer Systems III, Lecture Notes in Computer Science, Vol. 3279/2005, pp. 217-226, 2005.
[8] K. P. Gummadi, R. J. Dunn, S. Saroiu, S. D. Gribble, H. M. Levy, J. Zahorjan, "Measurement, Modeling, and Analysis of a Peer-to-Peer File-sharing Workload", Proc. of 19-th ACM Symposium on Operating Systems Principles, pp. 314-329, ACM Press, New York, NY, USA, 2003.

[9] CALAT Project, Nippon Telegraph and Telephone Corporation, http://www.calat.com/.

[10] CALsurf, NTT Software Corporation, http://webbase. ntts.co.jp/.

[11] The University of The Air, http://www. u-air.ac.jp/.

[12] California Virtual University, http://www.california. edu/.

[13] WIDE University, School of Internet, http://www. sfc.wide.ad.jp/soi/contents.html.

[14] Blacboard, http://www.blackboard.com/webct.

[15] L. Barolli, A. Koyama, "A Web-based Distance Learning System Using Cooperative Agents", Chapter in Encyclopedia of Online Learning and Technology, Idea Group Inc. Publishing, Vol.4, pp.430-439, April 2005.

[16] L. Barolli, A. Koyama, A. Durresi, G. De Marco, "A Web-based E-learning System for Increasing Study Efficiency by Stimulating Learner Motivation”, Journal of Information Systems Frontiers, Springer Publishers, Vol. 8, No. 4, pp. 297-306, September 2006.

[17] D. Brookshier, D. Govoni, N. Krishnan, J.C Soto, "JXTA: Java P2P Programming", Sams Publishing, 2002. 